\title{
Learning from the past and predicting the future
}

This issue of The Journal of Laryngology \& Otology looks both backwards and forwards. In a remarkable article, Spencer documents the work of Sir Harold Delf Gillies. ${ }^{1}$ It is a privilege to have the opportunity to publish an historical article of such quality. Spencer has been fortunate enough to gain access to the Gillies Archives, which is a record of the pioneering work that Gillies undertook in the reconstruction of major facial defects sustained by those who were mutilated during the 1914-18 First World War. The results achieved in these early reconstructive efforts were incredibly impressive, and the account of Gillies' life and work is testament to his status in the world of facial plastic and reconstructive surgery. Looking back even further, Kelly and Mahalingam explore the surgical treatment of head and neck cancers in the ancient world. ${ }^{2}$ They discover that a history of any aspect of Western medicine starts in antiquity and continues to the modern day. They discuss the state of medicine in Egyptian, Greek and Roman civilisations.

Looking now to the future, there has been much interest in the potential application of robotic surgery in the head and neck. ${ }^{3,4}$ Two articles in this issue examine the potential role of robotic surgical systems. Wang and colleagues undertook a meta-analysis of studies comparing robotic and conventional open thyroidectomy for differentiated thyroid cancer. ${ }^{5}$ They conclude that robotic thyroidectomy appears to be feasible and safe, although further evidence is needed. Another article in this issue describes the use of a surgical robotic system in the excision of a laryngeal haemangioma. ${ }^{6}$ There appear to be distinct advantages of this method of excision when compared to laser removal for this particular pathology.

As Editors, we are always prepared to publish 'Short Communications' that document innovation in surgical technique or novel solutions to long-established problems. In this issue, we publish four such articles. Of particular note is a suturing device for use in the nasal septum described by Kuboki and colleagues. ${ }^{7}$ It is interesting to read this article against an article from The Journal archives by Arnold Maran in which the author promotes the techniques of septoplasty as an alternative to classical submucosal resection. ${ }^{8}$

Finally, an article in this issue documents the apparent increase in incidence of necrotising otitis externa in the UK. ${ }^{9}$ This increase seems to be related to an increasing elderly population, and the increase in the incidence of diabetes. This condition is frequently clinically challenging, ${ }^{10,11}$ and clinical teams may have to devote more time to the management of affected patients.

\section{ROBIN YOUNGS EDWARD FISHER Senior Editors}

References

1 Spencer CR. Sir Harold Delf Gillies, the otolaryngologist and father of modern facial plastic surgery: review of his rhinoplasty case notes. J Laryngol Otol 2015;129:520-8

2 Kelly J, Mahalingam S. Surgical treatment of head and neck cancers in the ancient world. J Laryngol Otol 2015;129:535-9

3 Ciabatti PG, Burali G, D'Ascanio L. Transoral robotic surgery for large mixed laryngocoele. J Laryngol Otol 2013;127:435-7

4 Wang $\mathrm{C}-\mathrm{C}, \mathrm{Wu} \mathrm{C}-\mathrm{H}$. Non-recurrent inferior laryngeal nerve identification during robotic thyroidectomy. J Laryngol Otol 2014;128:199-202

5 Wang Y-C, Liu K, Xiong J-J, Zhu J-Q. Robotic thyroidectomy versus conventional open thyroidectomy for differentiated thyroid cancer: meta-analysis. J Laryngol Otol 2015;129: 558-67

6 Wang W-H, Tsai K-Y. Transoral robotic resection of an adult laryngeal haemangioma and review of the literature. J Laryngol Otol 2015;129:614-8

7 Kuboki A, Yanagi K, Nakayama T, Haruna S. Simple suturing of the nasal septum using the Maniceps septum stitch device. J Laryngol Otol 2015;129:591-4

8 Maran AG. Septoplasty. J Laryngol Otol 1974;88:393-405

9 Chawdhary G, Liow N, Democratis J, Whiteside O. Necrotising (malignant) otitis externa in the UK: a growing problem. Review of five cases and analysis of national Hospital Episode Statistics trends. J Laryngol Otol 2015;129:600-3

10 Spielmann PM, Yu R, Neeff M. Skull base osteomyelitis: current microbiology and management. J Laryngol Otol 2013; 127(suppl 1):S8-12

11 Lambor DV, Das CP, Goel HC, Tiwari M, Lambor SD, Fegade MV. Necrotising otitis externa: clinical profile and management protocol. J Laryngol Otol 2013;127:1071-7 